\title{
Operation of the ATLAS Transition Radiation Tracker under very high irradiation at the CERN LHC
}

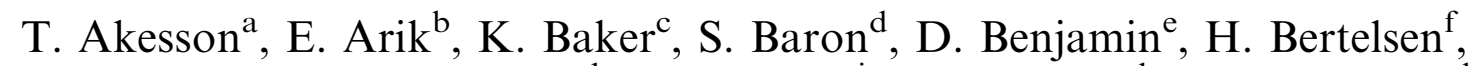
V. Bondarenko ${ }^{g}$, V. Bytchkov ${ }^{\mathrm{h}}$, J. Callahan ${ }^{\mathrm{i}}$, M. Capeans ${ }^{\mathrm{d}}$, L. Cardiel-Sas ${ }^{\mathrm{d}}$,

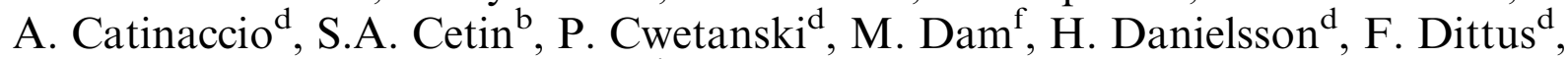
B. Dolgoshein ${ }^{\mathrm{g}}$, N. Dressnandt ${ }^{\mathrm{j}}$, C. Driouichi ${ }^{\mathrm{a}}$, W.L. Ebenstein ${ }^{\mathrm{e}}$, P. Eerola $^{\mathrm{a}}$, P. Farthouat ${ }^{\mathrm{d}}$, O. Fedin ${ }^{\mathrm{k}}$, D. Froidevaux ${ }^{\mathrm{d}}$, P. Gagnon ${ }^{\mathrm{i}}$, Y. Grichkevitch ${ }^{1}$, N. Grigalashvilih ${ }^{\text {h }}$ Z. Hajduk ${ }^{\mathrm{m}}$, P. Hansen ${ }^{\mathrm{f}}$, F. Kayumov ${ }^{\mathrm{n}, \mathrm{i}}$, P.T. Keener ${ }^{\mathrm{j}}$, G. Kekelidze ${ }^{\mathrm{h}}$, A. Khristatchev ${ }^{\mathrm{k}}$, S. Konovalov ${ }^{\mathrm{n}}$, L. Koudine ${ }^{\mathrm{k}}$, S. Kovalenko ${ }^{\mathrm{k}}$, T. Kowalski ${ }^{\circ}$, V.A. Kramarenko', K. Kruger ${ }^{d}$, A. Laritchev', P. Lichard ${ }^{\mathrm{d}}$, F. Luehring ${ }^{\mathrm{i}}$, B. Lundberg ${ }^{\mathrm{a}}$, V. Maleev ${ }^{\mathrm{k}}$, K. McFarlane ${ }^{\mathrm{c}}$, V. Mialkovski ${ }^{\mathrm{h}}$, B. Mindur ${ }^{\mathrm{O}}$, V.A. Mitsou ${ }^{\mathrm{d}}$, S. Morozov ${ }^{\mathrm{g}}$, A. Munar ${ }^{\mathrm{j}}, \mathrm{S}_{\text {. Muraviev }}^{\mathrm{n}}$, A. Nadtochy ${ }^{\mathrm{k}}$, F.M. Newcomer ${ }^{\mathrm{j}}$, H. Ogren ${ }^{\mathrm{i}}$, S.H. Oh ${ }^{\mathrm{e}}$, S. Oleshko ${ }^{\mathrm{k}}$, J. Olszowska ${ }^{\mathrm{m}}$, S. Passmore ${ }^{\mathrm{d}}$, S. Patritchev ${ }^{\mathrm{k}}$, V. Peshekhonov ${ }^{\mathrm{h}}$, R. Petti ${ }^{\mathrm{d}}$, M. Price ${ }^{\mathrm{d}}$, C. Rembser ${ }^{\mathrm{d}}$, O. Rohne, A. Romaniouk ${ }^{\mathrm{d}, \mathrm{g}, *}$, D.R. Rust ${ }^{\mathrm{i}}$, Yu. Ryabov $^{\mathrm{k}}$, V. Schegelsky $^{\mathrm{k}}$, D. Seliverstov ${ }^{\mathrm{k}}$,

T. Shin ${ }^{c}$, A. Shmelevan ${ }^{n}$, S. Smirnov ${ }^{g}$, V. Sosnovtsev ${ }^{g}$, V. Soutchkov ${ }^{\text {, }}$,

E. Spiridenkov ${ }^{\mathrm{k}}$, V. Tikhomirov ${ }^{\mathrm{n}}$, R. Van Berg ${ }^{\mathrm{j}}$, V. Vassilakopoulos ${ }^{\mathrm{c}}$, L. Vassilieva ${ }^{\mathrm{n}}$, C. Wang ${ }^{\mathrm{e}}$, H.H. Williams ${ }^{\mathrm{j}}$, A. Zalite ${ }^{\mathrm{k}}$

\author{
${ }^{a}$ Fysiska Institutionen, Lunds Universitet, Lund, Sweden \\ ${ }^{\mathrm{b}}$ Department of Physics, Bogazici University, Istanbul, Turkey \\ ${ }^{\mathrm{c}}$ Hampton University, Hampton, Virginia, USA \\ ${ }^{\mathrm{d}}$ European Laboratory for Particle Physics (CERN), EP Division, CH-1211 Geneva 23, Switzerland \\ ${ }^{\mathrm{e}}$ Physics Department, Duke University, Durham, NC, USA \\ ${ }^{\mathrm{f}}$ Niels Bohr Institute, University of Copenhagen, Copenhagen, Denmark \\ ${ }^{\mathrm{g}}$ Moscow Engineering and Physics Institute, Moscow, Russia \\ ${ }^{\mathrm{h}}$ Joint Institute of Nuclear Research, Dubna, Russia \\ ${ }^{\mathrm{i}}$ Department of Physics, Indiana University, Bloomington, IN, USA \\ ${ }^{\mathrm{j}}$ Department of Physics and Astronomy, University of Pennsylvania, Philadelphia, PA, USA \\ ${ }^{\mathrm{k}}$ Petersburg Nuclear Physics Institute, Gatchina, St. Petersburg, Russia \\ ${ }^{1}$ Moscow State University, Institute of Nuclear Physics, Moscow, Russia \\ ${ }^{\mathrm{m}}$ Henryk Niewodniczanski Institute of Nuclear Physics, Cracow, Poland \\ ${ }^{\mathrm{n}}$ P.N. Lebedev Institute of Physics, Moscow, Russia \\ ${ }^{\circ}$ Faculty of Physics and Nuclear Techniques of the Academy of Mining and Metallurgy, Cracow, Poland
}

\footnotetext{
*Corresponding author. CERN, Geneve 23 CH 1211, Switzerland. Tel.: + 41-22-767-11-76; fax: +41-22-767-83-50.

E-mail address: anatoli.romaniouk@cern.ch (A. Romaniouk).
} 


\begin{abstract}
The ATLAS Transition Radiation Tracker (TRT) performance depends critically on the choice of the active gas and on its properties. The most important operational aspects, which have led to the final choice of the active gas for the operation of the TRT at the LHC design luminosity, are presented. The TRT performance expected at these conditions is reviewed, including pile-up effects at high luminosity.
\end{abstract}

(C) 2004 Elsevier B.V. All rights reserved.

PACS: $29.40 .-\mathrm{n} ; 29.40 . \mathrm{Gx}$

Keywords: TRD; TRT; Proportional chamber; Straw; Ageing; High rate

\section{Introduction}

The ATLAS Transition Radiation Tracker (TRT) is one of the three subsystems constituting the ATLAS Inner Detector: it combines charged-particle track reconstruction with electron-identification capability. Details of the TRT design and construction status are given in Ref. [1]. The TRT detector elements and front-end electronics are situated close to the proton-proton interaction point and the particle fluxes through the straws are therefore very high. After 10 years of LHC operation, the TRT straws are expected to accumulate up to $10 \mathrm{MRad}$ of ionising radiation dose and up to $2 \times 10^{14} \mathrm{n} / \mathrm{cm}^{2}$ in terms of neutron fluence. The straw counting rates will reach a level of up to $20 \mathrm{MHz}$ in the regions closest to the beam line, the ionising current density will peak at $0.15 \mu \mathrm{A} / \mathrm{cm}$, and the maximum total accumulated charge after 10 years of operation will be approximately $10 \mathrm{C}$ per of wire. The induced radioactivity of $\mathrm{Xe}$ stored in the detector will be about $8 \mathrm{mCi}$ : this will pose some constrains on the access and maintenance scenarios for the TRT.

The results of the extensive studies performed over the past 12 years to ensure the reliability and stable operation of the ATLAS TRT during 10 years of LHC operation have been published in Refs. [2-7] and are reviewed here together with the more recent work related to the final choice of active gas and its impact on the operation and performance of the TRT at the LHC.

\section{New TRT baseline gas mixture}

Detailed studies carried out within the TRT collaboration [2-8] with gas mixture, composed of $70 \% \mathrm{Xe}+20 \% \mathrm{CF}_{4}+10 \% \mathrm{CO}_{2}$, have shown that this gas mixture combines the advantages of an efficient transition radiation absorption, a short electron drift time, a wide operation plateau and minimum ageing effects. A multi-parameter optimisation of the gas-mixture composition and of the straw tube itself to obtain the best possible TRT performance under LHC operating conditions have led to a well-defined design of the TRT straw tube, which is a $4 \mathrm{~mm}$ diameter cylindrical proportional chamber with a Carbon loaded polyimide cathode and a gold-plated tungsten anode wire with a diameter of $30 \mu \mathrm{m}$ [3].

Electro-chemical reactions occurring in the straws operating under irradiation produce fluorine-based active species ( $\left.\mathrm{HF}, \mathrm{F}, \mathrm{F}_{2} \ldots\right)$ which, on one hand, may inhibit or remove Si-deposits from the anode wires, but on the other hand, under certain conditions, may attack the detector materials and cause damage [2]. This is particularly relevant for only Si-containing components of the TRT such as the glass wire-joint used in the barrel TRT to split the $1.4 \mathrm{~m}$ long wire into two parts [1].

Photographs of samples of the glass wire-joints before and after some time of operation under the LHC operating conditions with the original gas mixture are shown in Figs. 1 and 2. These show that, during irradiation, the glass is etched away and the wire-joint eventually breaks and frees the wire. After almost one year of intensive $R \& D$ on 


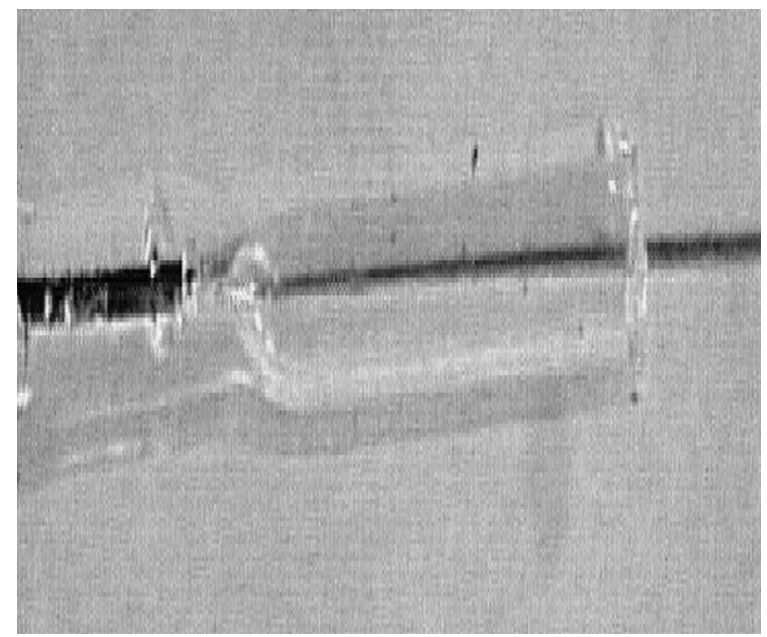

Fig. 1. Photograph of a glass wire-joint before irradiation.

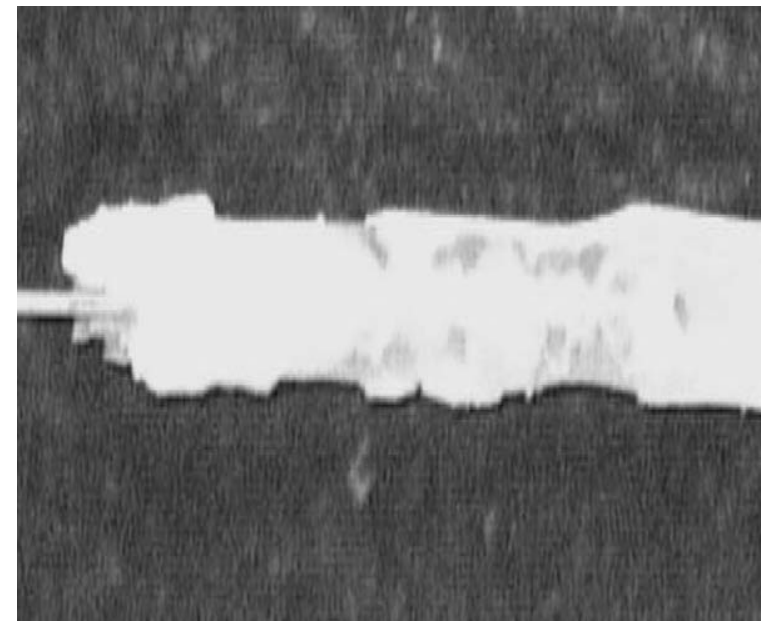

Fig. 2. Photograph of a glass wire-joint after irradiation.

alternate wire-joint technologies and alternate gas mixtures, it was decided to remove the $\mathrm{CF}_{4}$ from the active gas mixture.

A new gas mixture is required to have similar properties to the original one, but it should not contain components prone to the production of chemically aggressive radicals or polymerisation. A binary $\mathrm{Xe} / \mathrm{CO}_{2}$ mixture would be the simplest solution. However, such a mixture does not have a long enough operational plateau for the TRT, if no additional components are added to the mixture. Hydrocarbon gases are often used to stabilise the amplification process, but are prone to polymerisation on the anode wire (see below). One of the hydrocarbon candidates, however, is ethane, which is known in some cases to not produce polymerisation at relatively high radiation doses [9]. For the non-organic components, the choice is limited: after some research, oxygen was found to be the best candidate additive to the $\mathrm{Xe} / \mathrm{CO}_{2}$ for the TRT. Fig. 3 shows the difference between the working-point voltage (operational plateau) and the breakdown voltage as a function of the wire offset for different gas mixtures. For the TRT, the largest possible wire offset is $400 \mu \mathrm{m}$, since wires with larger offsets will be disconnected from high voltage before installation into ATLAS [1]. Fig. 3 clearly demonstrates that the addition of about $3 \%$ of ethane or oxygen improves substantially the operational stability of the gas mixture.

A critical issue for the choice of the new gas mixture was the compatibility of the front-end electronics design with the shape of the signal from the straw. This was of particular importance for operation at high counting rates, because preserving as much as possible the straw drift-time accuracy and efficiency requires a precise cancellation of the $\log$ ion tail. Fig. 4 shows the measured straw signal amplitude versus time for different gas mixtures. The ion tail for the original gas mixture

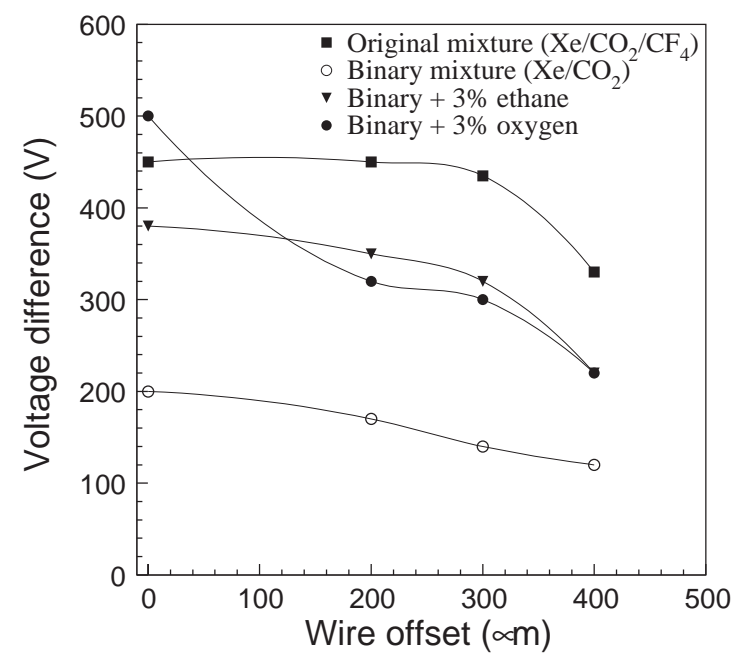

Fig. 3. Difference between the TRT straw working-point and breakdown voltages as a function of the wire offset for different gas mixtures. 


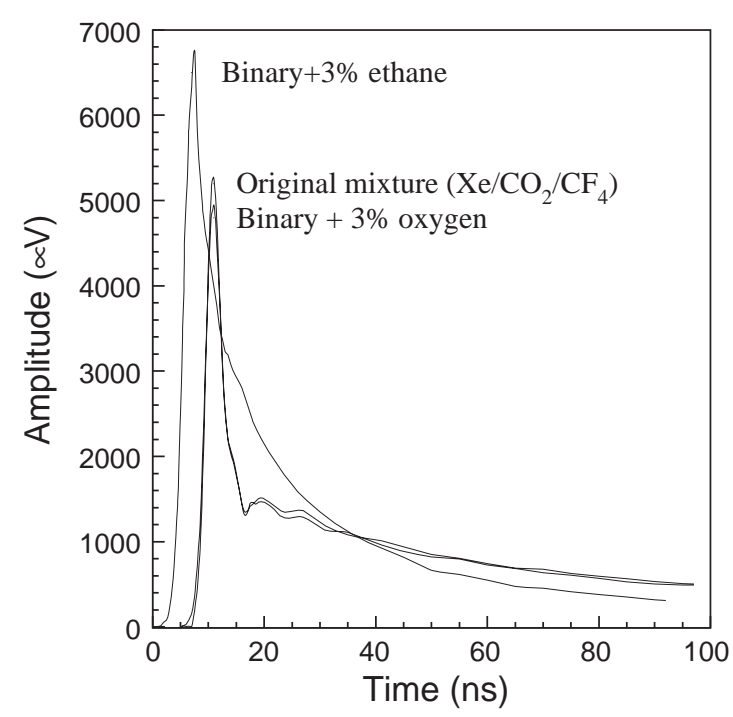

Fig. 4. Comparison of the straw signal amplitudes versus time for the TRT original gas mixture and for gas mixtures containing $3 \%$ of ethane and $3 \%$ of oxygen. The fast component of the signal for the original gas mixture is higher in amplitude than that for the binary mixture with $3 \%$ oxygen.

and for the $70 \% \mathrm{Xe}+27 \% \mathrm{CO}_{2}+3 \% \mathrm{O}_{2}$ mixture are exactly the same, with, however, a $7 \%$ decrease of the fast electron component of the signal in the case of the new gas mixture. The straw signal shape for the mixture containing ethane is however very different.

In addition to the fact that the shape of the signal substantially changes, definite ageing phenomena observed with this gas mixture makes its use impossible for the TRT at the LHC. This is demonstrated in Fig. 5. Forty $\mathrm{cm}$ long straw irradiated by a $10 \mathrm{~cm}$ wide beam (the irradiated region in Fig. 5 is between 150 and $250 \mathrm{~mm}$ ) with a gas flow of $0.15 \mathrm{~cm}^{3} / \mathrm{min}$ (gas-flow direction is from left to right in Fig. 5), and with a current density of $0.1 \mu \mathrm{A} / \mathrm{cm}$. After $30 \mathrm{~h}$ of irradiation, a significant amplitude drop was observed starting from the position of $180 \mathrm{~mm}$ along the straw. These results, followed by other studies, show that the ethane itself is not prone to polymerisation, but that the ionisation creates non-saturated hydrocarbons compound, which under certain concentrations (which increase from left to right in Fig. 5) produce deposits on the anode wire.

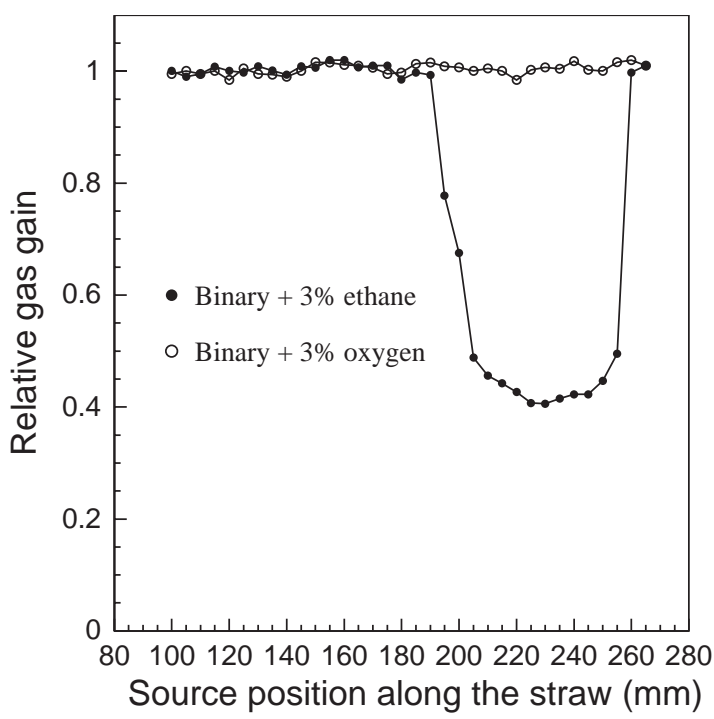

Fig. 5. Relative amplitude variation along a $40 \mathrm{~cm}$ long straw after $30 \mathrm{~h}$ of operation under irradiation with a $70 \% \mathrm{Xe}+$ $27 \% \mathrm{CO}_{2}+3 \%$ ethane gas mixture (see text). The irradiated area is from 150 to $250 \mathrm{~mm}$. The amplitude loss is removed after $18 \mathrm{~h}$ of operation with the new TRT gas mixture (see text).

Electron attachment to oxygen depends strongly on the electric field, since it is a maximum at thermal energies of the drifting electrons. This effect was studied in detail, and it was shown that the TRT performance is not affected if the $\mathrm{O}_{2}$ concentration is below $3.5-4 \%$. For the choice of a new gas mixture, $3 \%$ is sufficient to provide good operational stability (see Fig. 3). In fact oxygen itself is transparent to UV photons and cannot really improve the operational stability. The real improvement is provided by the ozone molecules, which are created in the avalanche near the wire. At high radiation doses, this ozone accumulates inside the straws, thereby increasing the operational plateau by at least a factor of two with respect to the one shown in Fig. 3 for the new gas mixture.

Ozone is, however, even more electronegative than oxygen and its accumulation leads to a degradation of the signal amplitude along the straw. For the barrel straws of $140 \mathrm{~cm}$ length, at a current density of $0.125 \mu \mathrm{A} / \mathrm{cm}$ (close to maximum at the LHC), the signal amplitude drops by about $13 \%$ over the first $50 \mathrm{~cm}$ of the straw length 
and then remains stable. This shows that the processes of ozone creation and dissociation under irradiation reach equilibrium at that point along the straw. The actual ozone concentration corresponding to this amplitude drop is about $150 \mathrm{ppm}$. Amplitude variations below $20 \%$ does not have any significant impact on the TRT performance. Additional long-term tests demonstrated that the detector compounds are not damaged by the presence of ozone in these quantities.

The new gas mixture with $3 \% \quad \mathrm{O}_{2}$ has very strong cleaning properties in the case of hydrocarbon deposits as shown in Fig. 5. After $18 \mathrm{~h}$ of operation with an oxygen-containing mixture, at a current density of $0.1 \mu \mathrm{A} / \mathrm{cm}$, the signal amplitude in the aged area was fully restored.

Removing $\mathrm{CF}_{4}$ from the TRT active gas mixture has other consequences. One of them is an increase of the electron collection time (see Section 3). Another one is an increase of the TRT sensitivity to Si-containing impurities. Although all possible measures are being taken to minimise the risk of introducing such impurities into the system, one cannot over 10 years of LHC operation, guarantee at $100 \%$ that this will not happen in a large and complex system such as the TRT. In order to preserve TRT operation in case of such occupancies a wire-cleaning procedure has been workedout. This actually relies on of operating the TRT by flushing it with an $\mathrm{Ar} / \mathrm{CO}_{2} / \mathrm{CF}_{4}$ mixture for a few days each year at the beginning or at the end of the LHC run. It was shown that 2-3 days of operation with this mixture removes Si-deposits from the wires, which had caused a $30 \%$ amplitude drop in the straw gas gain. A special study using this mixture has shown that this procedure is not dangerous for the glass wire-joint, if repeated once per year for a period of 10 years.

\section{TRT performance at the LHC conditions}

As described in Section 2, the straw signals for the original and new gas mixtures are almost identical and one should not expect a substantial change of the TRT performance at high rates using the same front-end electronics (TRT ASDBLR chip [1,7]). Figs. 6 and 7 show,

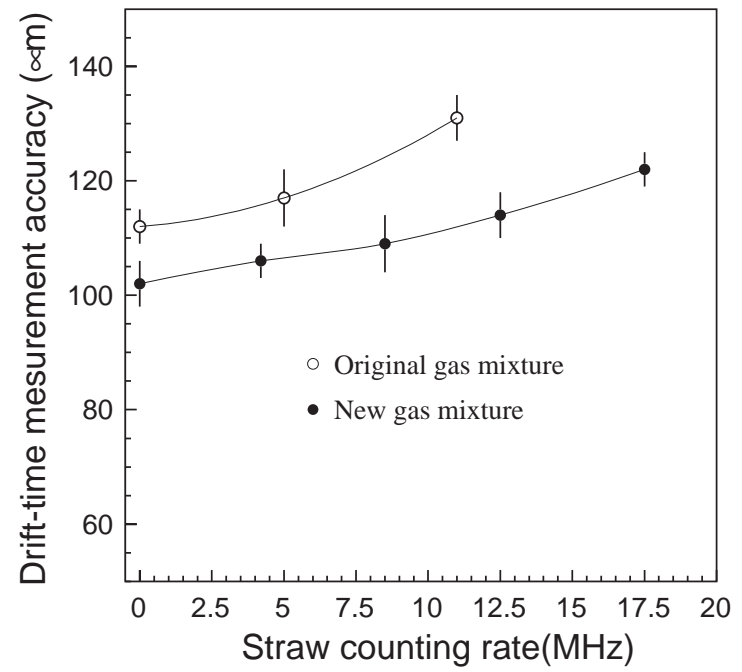

Fig. 6. Drift-time measurement accuracy as a function of the straw counting rate for the original $\left(\mathrm{Xe} / \mathrm{CF}_{4} / \mathrm{CO}_{2}\right)$ and new $\left(\mathrm{Xe} / \mathrm{CO}_{2} / \mathrm{O}_{2}\right)$ gas mixtures.

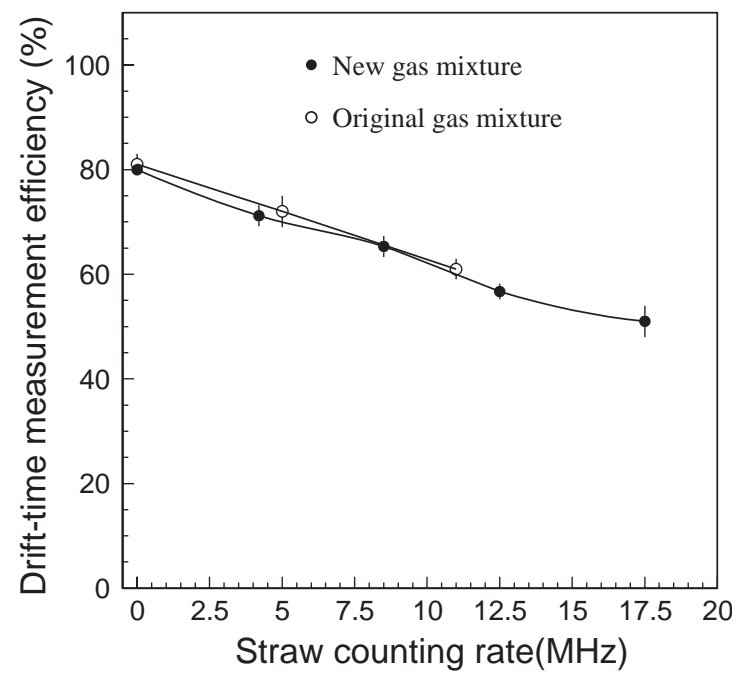

Fig. 7. Drift-time measurement efficiency as a function of the straw counting rate for the original $\left(\mathrm{Xe} / \mathrm{CF}_{4} / \mathrm{CO}_{2}\right)$ and new $\left(\mathrm{Xe} / \mathrm{CO}_{2} / \mathrm{O}_{2}\right)$ gas mixtures.

respectively, the drift-time measurement accuracy and efficiency for the original and new gas mixtures as a function of the straw counting rate. One sees that the drift-time measurement accuracy for the new gas mixture is by $10-20 \mu \mathrm{m}$ better than for the original one and that the efficiency is the same even at the highest counting rates expected at 
the LHC. The main reason for the worse drift-time accuracy in the original mixture seems to be large fluctuation of the signal from the arriving electron clusters due to very strong electron attachment in $\mathrm{CF}_{4}$ dissociation processes at high electric fields.

High particle densities, such as those of up to $500 \mathrm{kHz} / \mathrm{cm}$ expected in the most exposed parts of the TRT straws at the LHC, may induce space charge effects, which can affect the TRT performance. These effects have been studied in detail for the original TRT gas mixture [3]. No change was observed for the signal amplitude up to $500 \mathrm{kHz} / \mathrm{cm}$ and for the drift-time accuracy up to $750 \mathrm{kHz} / \mathrm{cm}$.

These studies were repeated for the new gas mixture. A straw exposed to test-beam particles was irradiated at the same time by a $6 \mathrm{keV} \mathrm{X}$-ray source with a maximum particle rate of $350 \mathrm{kHz} / \mathrm{cm}$ of wire. The space-charge effects expected from X-rays at this rate correspond to those expected from minimum-ionising particles at a rate of $900 \mathrm{kHz} / \mathrm{cm}$ (about twice the maximum expected at the LHC). The straw drift-time accuracy degraded from 110 (0 rate) to $130 \mu \mathrm{m}$ (maximum rate) without any significant change of the drift-time efficiency itself. Since the straw drift-time accuracy has not been observed to be affected at these high rates for the original gas mixture, the degradation observed in these measurements is assumed to arise from the high ozone concentration in the avalanche region.

As already mentioned above, electrons in the new gas mixture have a smaller drift-velocity. The total electron collection time in the new gas mixture increases by $4 \mathrm{~ns}$ (in a magnetic field of $2 \mathrm{~T}$ ) with respect to the original gas mixture, thereby leading to an increase in the straw occupancy. The on detector electronics readout stores the information from the straws in three sequential time slices of $25 \mathrm{~ns}$ each [1], corresponding to three consecutive LHC bunch-crossings. A longer charge-collection time in the straw leads to larger overlap from adjacent bunches with the bunch-crossing of interest, and hence for larger straw occupancy. In order to reduce this pile-up occupancy from adjacent bunch-crossings, only straws exhibiting a signal in a certain narrow time interval (gate) are considered as valid straws for the analysis. Fig. 8 shows the distribution of low-threshold discriminator bits

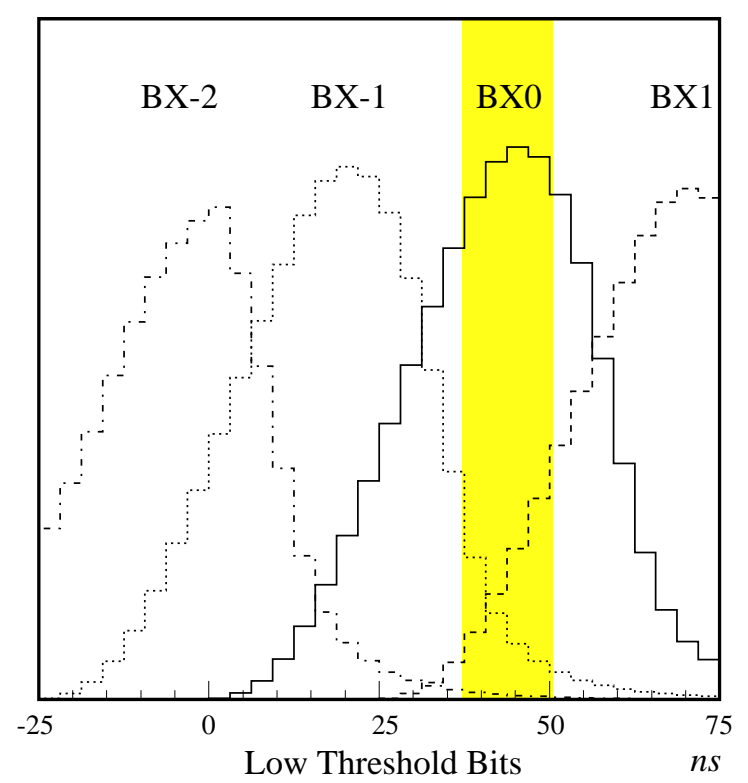

Fig. 8. Distribution of low-threshold discriminator bits fired for the four most important bunch-crossings, where BX0 designates the bunch-crossing of interest. The hatched area indicates the chosen $12.5 \mathrm{~ns}$ gate, which efficiently records valid straw hits and minimises the contribution from the adjacent bunch-crossings.

fired for the four most important bunch-crossings $(\mathrm{Bx}-2, \mathrm{BX}-1, \mathrm{BX} 0$ and $\mathrm{BX}+1)$, where $\mathrm{BX} 0$ designates the bunch-crossing of interest. The shaded area indicates the position of the $12.5 \mathrm{~ns}$ gate, which was found to be the narrowest time interval providing very efficient hit registration for the bunch-crossing of interest. An optimum choice of the position of this gate minimises the impact of pile-up from the adjacent bunch-crossings.

An illustration of the reduction of the pile-up from adjacent bunch-crossings using the method described above is shown in Figs. 9 and 10, which display in the transverse plane the hit straws in the part of the barrel TRT at the LHC design luminosity corresponding to an average of 23 minimum bias interactions per bunch crossing. Each point in these figures represents a straw with a hit within three time slices (Fig. 9) or within the chosen 12.5 ns gate (Fig. 10). The tracks generated in the bunch-crossing of interest are also shown and are much more clearly recognised when only valid straws are displayed. This method will be 


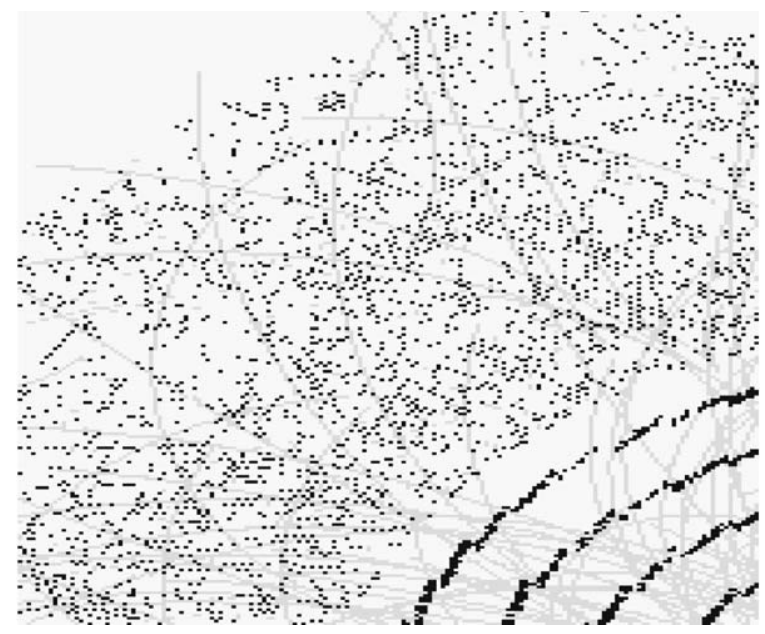

Fig. 9. Partial display in the transverse plane of a simulated event in the barrel TRT at the design luminosity corresponding to an average of 23 minimum-bias interaction per bunch crossing. Each point represents a straw with a hit within the three $25 \mathrm{~ns}$ time slices defined for the on-detector electronics readout.

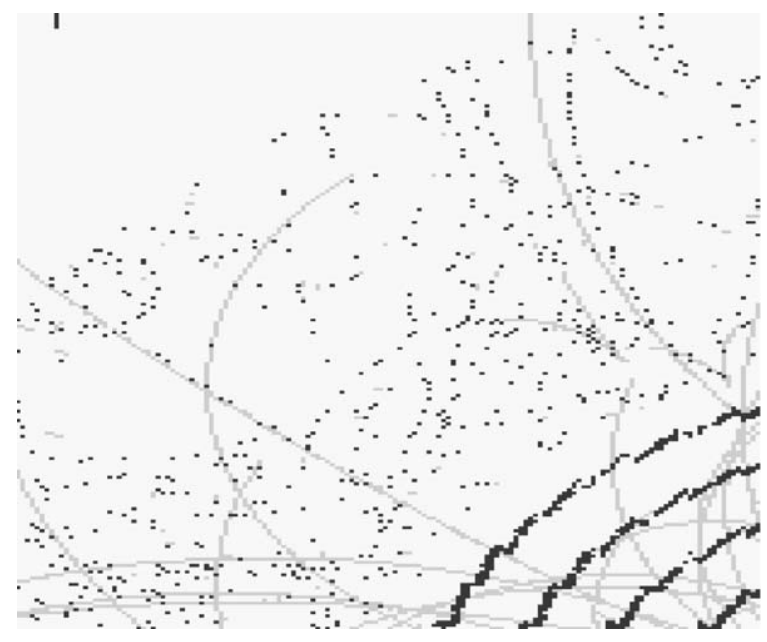

Fig. 10. The same as Fig. 9, after having applied the gate shown in Fig. 8 to minimise contribution from pile-up hits.

applied in the first readout stages and will therefore reduce substantially the data flow from the TRT detector, which is one of the largest in the ATLAS detector.

A comparison between the original and new gas mixtures showed that the occupancy, defined by this method, is $6 \%$ bigger for the new gas mixture.

\section{Conclusions}

The TRT detector will be operated at the LHC with a new $70 \% \mathrm{Xe} / 27 \% \mathrm{CO}_{2} / 3 \% \mathrm{O}_{2}$ gas mixture, which has recently been validated in terms of performance at high rate and of ageing properties. The removal of the $\mathrm{CF}_{4}$ component from the gas mixture has solved the difficult problem arising from severe damages caused to some of the TRT components (glass wire-joints in the barrel modules) due to active fluorine radicals produced under irradiation. The new gas mixture is however more sensitive to Si-ageing phenomena and $\mathrm{Ar} / \mathrm{CO}_{2} / \mathrm{CF}_{4}$ gas mixture will therefore be used periodically to clean the anode wires from any $\mathrm{Si}$ contaminations if the need arises.

Particular attention has been devoted to the assessment of the impact of ozone created in the avalanche process on the performance and operation of the detector. No damage to the TRT components has been observed from ozone. In the most difficult case of the long barrel straws, the expected ozone concentration is at most $150 \mathrm{ppm}$ and leads to at most $13 \%$ loss of straw signal amplitude, which does not significantly affect the TRT performance.

Finally, the new gas mixture provides $10 \%$ improvement in the drift-time accuracy but its smaller electron drift velocity yields a $6 \%$ increase in the straw occupancy from pile-up hits.

\section{Acknowledgements}

We are grateful to P. Nevski for his contributions to the-test beam and Monte-Carlo software development. The research described in this publication was partly supported by following funding agencies: the European Union (DGXII), the International Science Foundation (Grant NM5J000), the Swedish Natural Science Research Council, the Swedish Council for Planning and Coordination of Research, the State Committee for Scientific Research, Poland (Grant 620/E-77/ SPUB-M/CERN/P-03/DZ295/2000-2002), the International Science and Technology Centre (ISTC projects 441 and 1800P), the Civil Research and Development Foundation (Grant REC-011) and 
grants from U.S. Department of Energy and National Science Foundation.

\section{References}

[1] T. Akesson et al., Status of design and construction of the ATLAS TRT, Nucl. Instr. and Meth. A, (2004) these proceedings.

[2] T. Akesson, et al., Nucl. Instr. and Meth. A 515 (2003) 166.
[3] T. Akesson, et al., Nucl. Instr. and Meth. A 361 (1995) 440.

[4] T. Akesson, et al., Nucl. Instr. and Meth. A 367 (1995) 143.

[5] T. Akesson, et al., Nucl. Instr. and Meth. A 372 (1996) 70.

[6] T. Akesson, et al., Nucl. Instr. and Meth. A 412 (1998) 200.

[7] T. Akesson, et al., Nucl. Instr. and Meth. A 449 (2000) 446.

[8] A. Romaniouk, Workshop Proceedings TRDs for the Third Millennium, Barri, Italy, 20-23 September, 2001; Frascati Physics Series XXV (2001) 93.

[9] J. Vavra, Nucl. Instr. and Meth. A 252 (1986) 547. 